Marie Bartoušková, Michaela Hrouzková, Filip Čtvrtlík, Pavla Petrová, Nikol Rušarová, Kateřina Kučerová, Andrea Vernerová, Bohuslav Melichar, Hana Študentová*

\title{
Potential utilization of neopterin measurements in the assessment of pyrexia in metastatic melanoma treated with combined targeted therapy: a case report
}

https://doi.org/10.1515/pteridines-2020-0009

received April 13, 2020; accepted July 16, 2020.

\begin{abstract}
In patients with metastatic melanoma the advent of targeted therapy and immune checkpoint inhibitors has transformed the management of advanced and metastatic disease, resulting in improved outcomes. Neopterin is a biomarker of immune activation increased in cancer as well as in other conditions associated with immune activation. We present a case of a patient with advanced metastatic melanoma responding to the combination targeted therapy with dabrafenib and trametinib. The treatment was complicated by a fever that was accompanied by a marked rise in serum and urinary neopterin concentrations. Present case report illustrates not only the efficacy of combined targeted therapy, but also the utilization of neopterin measurements in the diagnosis and monitoring of pyrexia in patients with metastatic malignant melanoma.
\end{abstract}

Keywords: metastatic melanoma; neopterin; BRAF inhibitors; MEK inhibitors.

\footnotetext{
*Corresponding author: Hana Študentová, Department of Oncology, Faculty of Medicine and Dentistry and University Hospital, Palacký University Olomouc, Czech Republic, E-mail: hana.studentova@fnol.cz Marie Bartoušková, Michaela Hrouzková, Nikol Rušarová, Bohuslav Melichar, Department of Oncology, Faculty of Medicine and Dentistry and University Hospital, Palacký University Olomouc, Czech Republic

Filip Čtvrtlík, Department of Radiology, Faculty of Medicine and Dentistry and University Hospital, Palacký University Olomouc, Czech Republic

Pavla Petrová, Department of Clinical Biochemistry, Faculty of Medicine and Dentistry and University Hospital, Palacký University Olomouc, Czech Republic

Kateřina Kučerová, Andrea Vernerová, Department of Analytical Chemistry, Faculty of Pharmacy, Charles University, Hradec Králové, Czech Republic
}

\section{Introduction}

Malignant melanoma is a heterogeneous cluster of neoplastic disorders characterized by a variable and unpredictable biological behavior. Cases of melanoma can be distinguished according to the site of the primary tumor and molecular pathogenesis. About 90\% of melanoma cases originate in the skin, and about half of skin melanomas harbor an activating BRAF mutation [1]. Before the advent of targeted therapy and immune checkpoint inhibitors, the prognosis of patients with metastatic melanoma was almost uniformly dismal as cytotoxic chemotherapy and cytokines have been shown to be only marginally active $[2,3]$.

Ipilimumab, a monoclonal antibody targeting the cytotoxic T- lymphocyte antigen (CTLA-4) was the first drug shown to prolong survival in metastatic melanoma [4]. However, the response rate with ipilimumab monotherapy was relatively low, and has been quickly surpassed by the outcomes obtained with monoclonal antibodies targeting programmed death receptor (PD)-1 pembrolizumab and nivolumab [5, 6]. Subsequently, superior activity of the combination of ipilimumab and nivolumab over immune checkpoint inhibitor monotherapy has been demonstrated [6].

The development of new therapeutics in malignant melanoma also followed a parallel path. Approximately $50 \%$ of cases of cutaneous melanoma are characterized by the V600 mutation of the BRAF gene $[1,7]$. In patients with BRAF V600 mutation-positive melanoma, targeted inhibition of mitogen-activated protein kinases (MAPK) pathway results in rapid tumor regression. Combination of a BRAF inhibitor (dabrafenib, vemurafenib, or encorafenib) with a MEK inhibitor (trametinib, cobimetinib, or binimetinib) reduces the risk of acquired resistance and decreases the toxicity in comparison with single agent BRAF inhibition [8-10]. Adverse events of BRAF inhibitors include fever (dabrafenib), photosensitivity 
(vemurafenib), skin rash, or electrocardiographic changes (QTc prolongation).

Laboratory medicine plays a crucial role in the management of cancer patients [11]. In malignant melanoma the determination of the presence of BRAF mutation is essential for targeted therapy. However, less attention has been focused on the laboratory biomarkers of toxicity associated with the therapy. A notable side effect of the treatment with dabrafenib and trametinib combination is pyrexia that has to be distinguished from fever of other origin [12-18].

Neopterin is a biomarker of immune response produced by macrophages activated by interferon-gamma $[19,20]$. Neopterin is increased in patients with advanced cancer [19, 20], including malignant melanoma [21, 22], and neopterin concentrations may further increase after treatment with chemotherapy, cytokines and radiotherapy [20, 23]. Moreover, increased neopterin concentrations are associated with poor prognosis [24].

We present here a case of a patient with remarkable response to the combination of dabrafenib and trametinib accompanied by marked changes in serum and urinary neopterin concentrations that reflected the course of the disease and therapy, in particular the fever induced by the treatment.

Ethical approval: The research related to human use has been complied with all the relevant national regulations, institutional policies and in accordance the tenets of the Helsinki Declaration, and has been approved by the authors' institutional review board or equivalent committee.

Informed consent: Informed consent has been obtained from the patient included in this study

\section{Case Description}

A 47-year old woman was admitted to a regional hospital on June 12, 2014 because of life threatening grade 4 anemia (hemoglobin concentration of $25 \mathrm{~g} / \mathrm{L}$ ) caused by hemorrhage from ulcerated tumor in the left groin. The patient was transfused five red blood cell units and received intravenous fluids. A biopsy from the left groin established the diagnosis of malignant melanoma metastasis. Eight days later the patient was transferred to the Department of Oncology, University Hospital Olomouc, Czech Republic. Upon admission, blood pressure of $110 / 65 \mathrm{~mm} \mathrm{Hg}$, heart rate of 95 per minute, and Eastern Cooperative Oncology Group (ECOG) performance status of 4 were recorded. Lymphedema of the left lower extremity and an ulcerated melanoma of 5 centimeters in

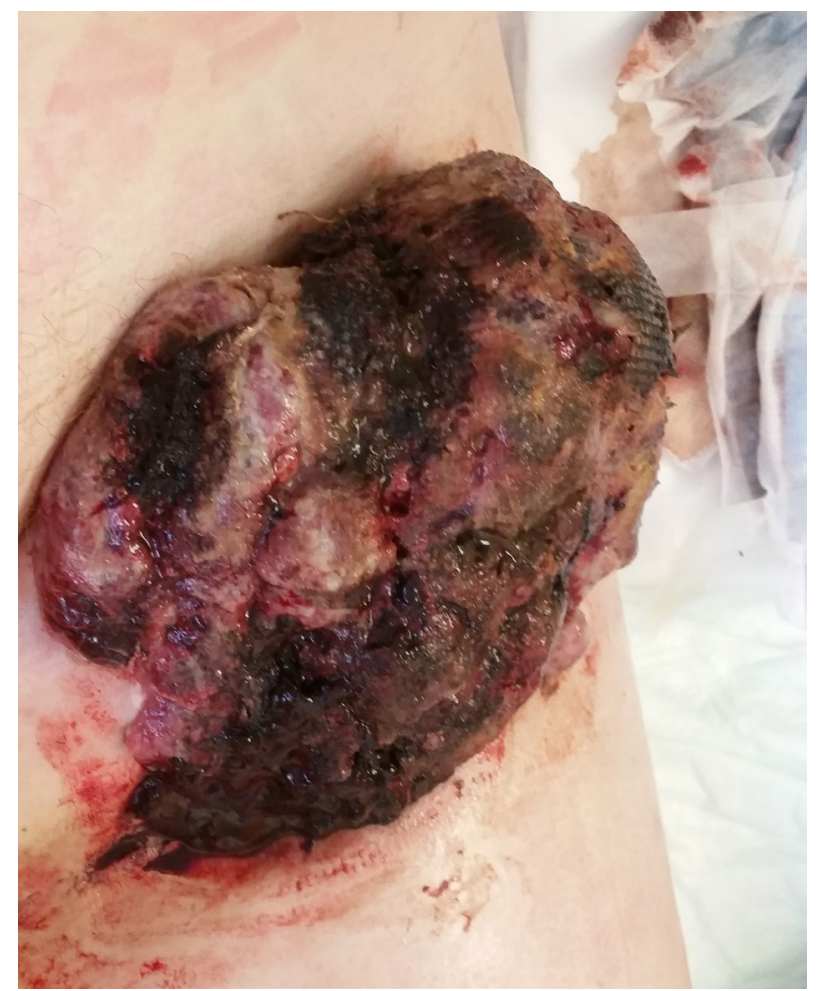

Figure 1: Ulcerated metastasis in the left groin at the start of the treatment.

diameter on the ventral side of the left calf were noted. In the left groin ulcerated metastasis of total size of 15 centimeters with spontaneous bleeding and necrosis was evident (Figure 1). Laboratory test showed a grade 2 anemia (hemoglobin concentration of $84 \mathrm{~g} / \mathrm{L}$ ), and high concentration of C-reactive protein (CRP; $104 \mathrm{mg} / \mathrm{L}$ ). Tumor BRAF mutation was detected. Supportive care, including daily dressing of the ulcerated metastasis, nutritional support, and red blood cell transfusion were provided. Subsequent computed tomography (CT) scan confirmed an ulcerated solid tumor of 163 x 105 x $150 \mathrm{~mm}$ in the left groin, a metastasis to the left ovary of a total size of $100 \times 65 \mathrm{~mm}$, and partial thrombosis of the right femoral vein (Figure $2 \mathrm{~A}$ and B). On July 4, 2014, because of rapid tumor progression and confirmed presence of BRAF mutation, combined targeted therapy with dabrafenib (150 mg twice daily) plus trametinib (2 mg once daily) was started. Samples for urinary neopterin determination were collected daily.

On July 28, 2014 ( $24^{\text {th }}$ day of the therapy) the condition of the patient suddenly deteriorated. The patient was confused and had fever above $40^{\circ} \mathrm{C}$. Laboratory test showed serum neopterin concentration above $28.10 \mu \mathrm{g} / \mathrm{L}$ and grade 3 anemia, but CRP concentration decreased $(65 \mathrm{mg} / \mathrm{L})$. The patient underwent brain CT scan, which excluded serious intracranial pathology. The time course 

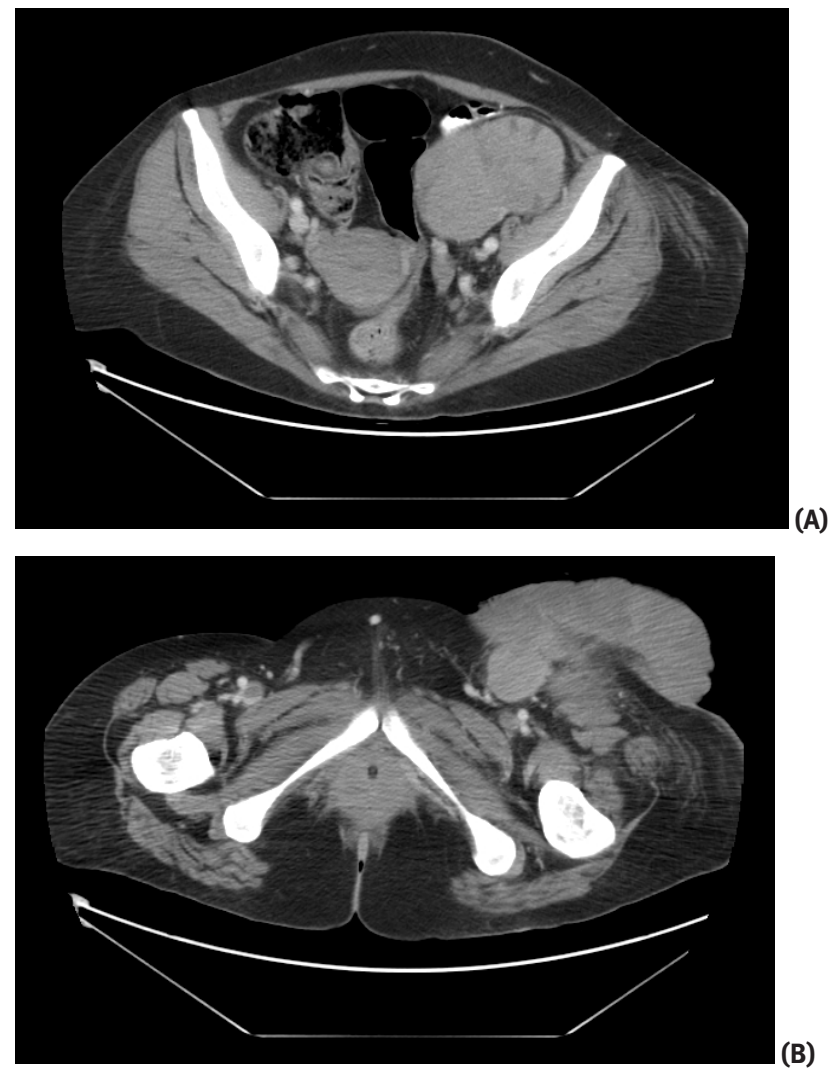

Figure 2: Computed tomography scans at the time of diagnosis. A metastasis to the left ovary (A), ulcerated solid tumor in the left groin (B), and partial thrombosis of the right femoral vein in the coronal plane are shown.

of serum neopterin, determined by immunoassay as described earlier [23] is shown in Figure $3 \mathrm{~A}$. The changes in urinary neopterin (Figure $3 \mathrm{~B}$ ) determined daily by high performance liquid chromatography [23] showed a similar trend, in contrast to serum CRP concentrations (Figure $3 \mathrm{C}$ ) determined by a commercial kit [25]. Because of the presentation, including rising neopterin concentration and decreasing CRP, and absence of any other general or organ-specific symptoms suggestive of infectious disease, the fever was considered a side effect of therapy, and not a manifestation of infection.

The results of daily urinary neopterin measurements indicate that after an initial decrease urinary neopterin concentrations began to rise 4 days after the start of therapy. Urinary neopterin concentrations were then stable for about two weeks at a higher level before another steep increase that began about a week before the manifestation of symptoms that coincided with peak neopterin concentration (Figure $3 \mathrm{~B}$ ).

Patient received hydratation, acetaminophen, corticosteroids (prednisone $10 \mathrm{mg}$ ), was transfused with three units of red blood cell for grade 3 anemia, and the targeted therapy was interrupted. Administration of antibiotic therapy (cefuroxime $500 \mathrm{mg}$ twice daily) that was initiated was considered a more or less prophylactic measure as the episode was considered to be noninfectious in face of declining CRP concentration. Two days later the patient condition improved, serum neopterin concentration decreased to $17 \mu \mathrm{g} / \mathrm{L}$, and targeted therapy was reinstituted. Urinary neopterin concentration also decreased markedly while the patient was recovering. Daily dressing including toilette and debridement of necrotic tissue was critical in preventing secondary infection of the ulcerated metastasis. It was also possible to monitor immediately the effect of the treatment. During course of hospitalization rapid and significant tumor shrinkage was observed accompanied by spontaneous shedding of the necrotic tumor (Figure 4). Intermittent pyrexia was the only adverse events of combined targeted therapy noted. During the period of daily urinary neopterin measurement another peak of increased concentration was observed (Figure $3 \mathrm{~B}$ ). Targeted therapy was otherwise well tolerated with rapid response. The patient was discharged and continued with combined targeted therapy on an outpatient basis.

In February 2015 control CT scan confirmed significant tumor regression (Figure $5 \mathrm{~A}$ and B). In April 2015 external beam radiation (total dose of 24 Gy in 3 fractions) was administered because of local progression in the left groin. In June 2015 the patient was admitted with thrombocytopenia, purpura and fever. Laboratory tests showed elevation of CRP (430.9 mg/L), acute kidney injury (urea $21.9 \mathrm{mmol} / \mathrm{L}$ and creatinine $353 \mu \mathrm{mol} / \mathrm{L}$ ), elevated level of lactate dehydrogenase ( $>30 \mu \mathrm{kat} / \mathrm{L})$, anemia grade $3(68 \mathrm{~g} / \mathrm{L})$, thrombocytopenia grade 3 (32 x $\left.10^{9} / \mathrm{L}\right)$, leukocytosis $\left(28.43 \times 10^{9} / \mathrm{L}\right)$, elevated levels of D-dimer $(247242 \mu \mathrm{g} / \mathrm{L})$. Antibiotic therapy was initiated immediately (cefotaxime, $1 \mathrm{~g}$ three times daily), and the patient was transfused with three red blood cell units. The two days later, on 12 June 2015 patient died supposedly of severe sepsis complicated by disseminated intravascular coagulation. An autopsy was not performed due to the known extensive metastatic disease.

\section{Discussion}

The present case report demonstrating the efficacy of combined targeted therapy in a patient with BRAF-mutated melanoma also illustrates the potential role of repeated neopterin measurements in the supportive care, including the diagnosis and monitoring of side effects of targeted therapy. During the past decade we have witnessed 

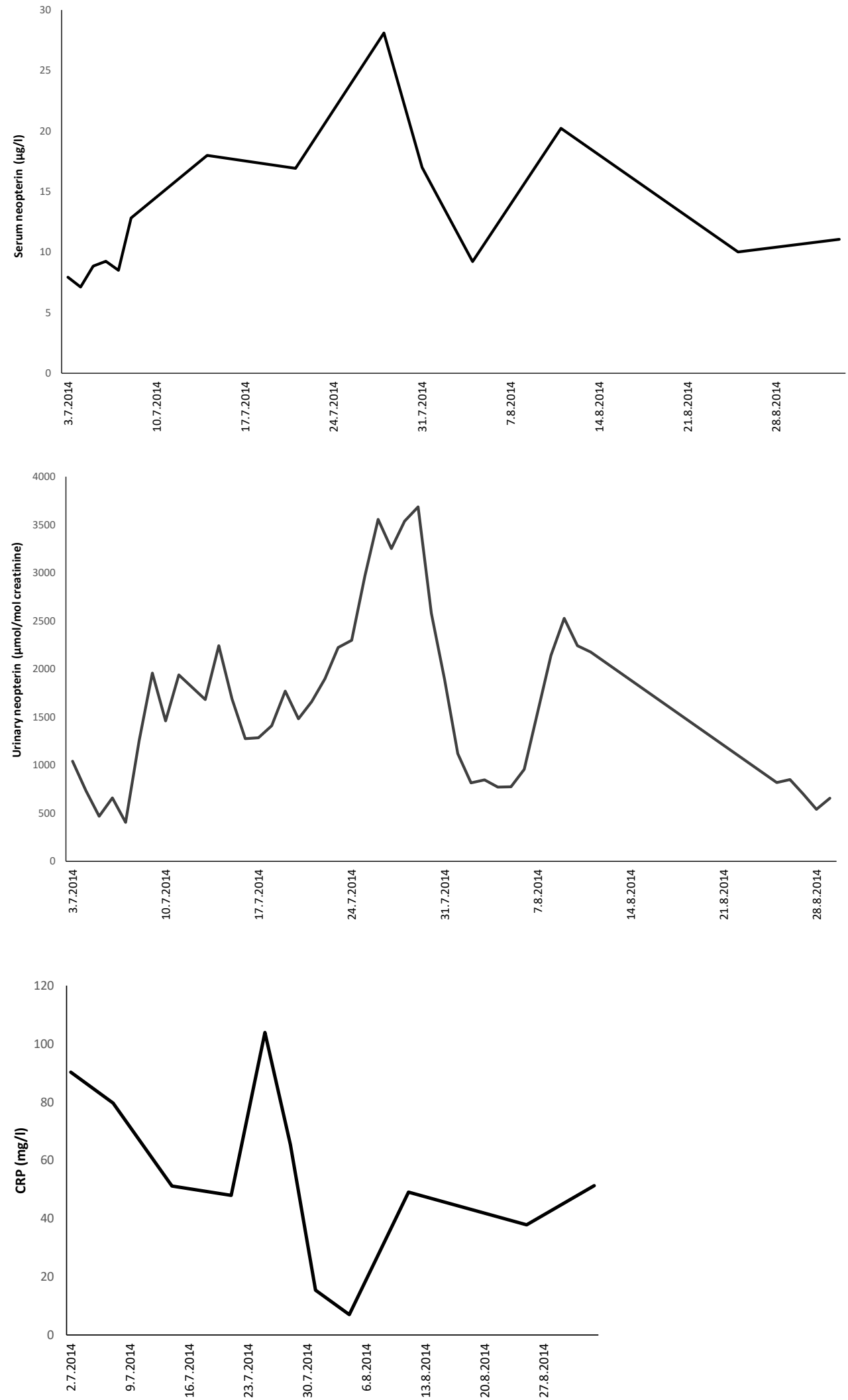

Figure 3: (A) Serum neopterin concentrations during the course of treatment. (B) Urinary neopterin concentrations during the course of treatment. (C) Serum C-reactive protein concentrations during the course of treatment. 




Figure 4: Shrinkage of the groin metastasis during the treatment.
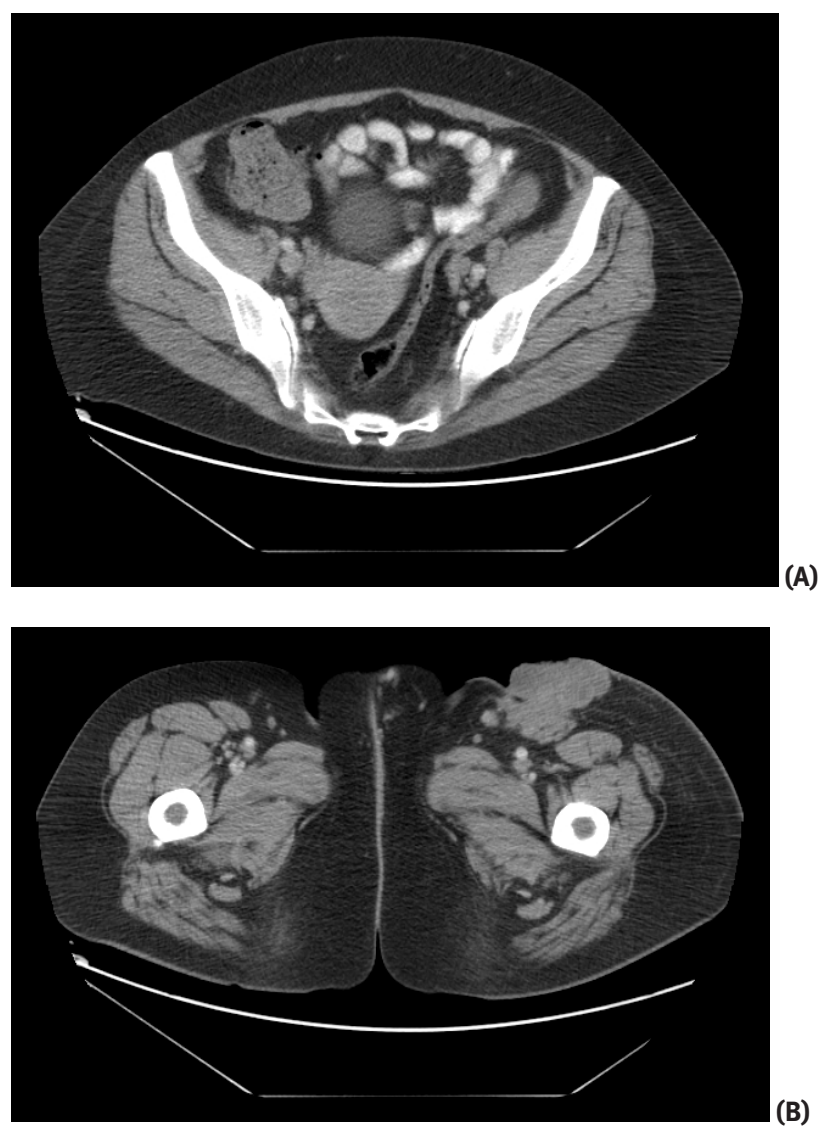

Figure 5: Regression of the disease after targeted therapy in February 2015. Compared to baseline (Figure 2) a partial response was evident.

a major paradigm shift in medical oncology with the introduction of targeted therapy. The advent of targeted therapy has virtually transformed the management of tumors resistant to radiation and chemotherapy like renal cell carcinoma $[26,27]$ and malignant melanoma [8]. Targeted therapy currently represents a treatment of choice for symptomatic patients with BRAF-mutated metastatic melanoma and high tumor burden as it induces rapid tumor shrinkage leading to the relief of symptoms. Unfortunately, most melanoma patients who initially respond to combined BRAF and MEK inhibition experience subsequently disease progression caused by acquired resistance. The other therapeutic option in this setting, the therapy with immune checkpoint inhibitors could result in long-term survival, but delayed onset of response or pseudo-progression may represent a problem in patients with high burden of metastatic disease as there may not be sufficient time for response manifestation.

Pyrexia with associated symptoms of chills, night sweats, dehydration or hypotension is the principal and most common side effect of the dabrafenib plus trametinib combination [18]. The mechanism of hyperpyrexia induced with dabrafenib and trametinib combination is still unknown $[15,16]$. It has been hypothesized that the cause of hyperpyrexia is paradoxical activation of MAPK pathway in lymphocytes, similarly to familial Mediterranean fever [16]. Present data demonstrate pyrexia in patients treated with dabrafenib plus trametinib is accompanied by markedly increased production of neopterin, a biomarker of immune response.

Biomarkers are essential for the management of cancer patients [11], and there is an unmet medical need for new biomarkers in patients treated with targeted therapy comprising immune checkpoint inhibitors, including patients with melanoma. Neopterin is a biomarker of immune activation, and increased neopterin concentrations were demonstrated in different disorders associated with immune activation, including viral infections, autoimmune diseases, myocardial infarction, or cancer [19, 28-30]. In cancer patients, increased neopterin production in the tumor microenvironment or systemically has been associated withimmune dysfunction [31-34]. It has been demonstrated that changing neopterin concentrations reflect the administration of therapy or complications [23].

In the present case report, daily monitoring of neopterin concentrations during the first two months of therapy was instrumental in distinguishing between fever accompanying bacterial infection and pyrexia caused by the therapy. The time course of daily measurements indicates that after an initial decline neopterin concentrations began to increase, indicating an activation of immune system. A further marked increase in urinary neopterin concentrations started about a week before the culmination of symptom manifestation at which time also urinary neopterin concentrations peaked. The supportive therapy resulted in a swift decrease in urinary neopterin that began to increase again after the re-institution 
of treatment. In the present case of a large ulcerated metastasis it was important to exclude infection as the cause of pyrexia. In this situation increasing urinary and serum neopterin concentrations while CRP level was decreasing suggested an etiology different from bacterial infection. Moreover, the presentation did not include, besides fever, any general or organ-specific symptoms suggestive of a bacterial or viral infection. The decrease of neopterin concentrations after institution of supportive therapy also indicated a non-infectious cause of the fever. Present data indicate that, in addition to aiding with the diagnosis, urinary neopterin concentration may be used to monitor as well as to predict fever associated with the administration of dabrafenib plus trametinib combination, but further studies are needed to investigate relationship between immune-related adverse events and neopterin concentration. Present data also suggest potential use of serial neopterin measurements for the assessment of the efficacy of therapeutic interventions in the supportive care of patients with pyrexia induced by combined BRAF and MEK inhibition.

With the advent of new drugs targeting pathogenic pathways of malignant transformation, including the evasion of the immune system [35], the spectrum of adverse events expands considerably [36]. While for cytotoxic agents the most prominent side effects are on rapidly dividing tissues, i.e. bone marrow and gastrointestinal mucosa [37], the spectrum of side effects of targeted agents is much wider and may often be caused by the activation of immune system [36]. In addition to immune checkpoint inhibitors, other targeted agents, e.g. the combination of dabrafenib with trametinib, could also activate the immune system. Patients with severe immune-related adverse events often require intensive care and multidisciplinary approach as this toxicity may be potentially fatal, and the present case report also illustrates importance of supportive treatment, including intensive care in medical oncology.

In conclusion, the present case reports demonstrating efficacy of the treatment with dabrafenib and trametinib also indicates potential use of daily neopterin measurements in the assessment of fever induced by this combination therapy, specifically the differentiation between the pyrexia induced by the treatment and fever of infectious origin and in the monitoring during the therapy. Conflict of Interest Statement: BM and HŠ; honoraria for speeches and advisory role Roche, Pfizer, BMS, Astellas, Novartis, Bayer, MSD, Merck Serono, Sanofi, Servier, AstraZeneca, Amgen, Janssen, Eisai, E. Lilly, Pierre Farbre. BM is the member of Pteridines Editorial Board.
Other authors declare no conflicts of interest regarding the publication of this article.

Data Availability Statement: The datasets generated during and/or analysed during the current study are available from the corresponding author on reasonable request.

\section{References}

1. Shinozaki M, Fujimoto A, Morton DL, Hoon DS. Incidence of BRAF oncogene mutation and clinical relevance for primary cutaneous melanomas. Clin Cancer Res. 2004 Mar;10(5):17537.

2. Atkins MB, Lotze MT, Dutcher JP, Fisher RI, Weiss G, Margolin K, et al. High-dose recombinant interleukin 2 therapy for patients with metastatic melanoma: analysis of 270 patients treated between 1985 and 1993. J Clin Oncol. 1999 Jul;17(7):2105-16.

3. Serrone L, Zeuli M, Sega FM, Cognetti F. Dacarbazine-based chemotherapy for metastatic melanoma: thirty-year experience overview. J Exp Clin Cancer Res. 2000 Mar;19(1):21-34.

4. Hodi FS, O'Day SJ, McDermott DF, Weber RW, Sosman JA, Haanen JB, et al. Improved survival with ipilimumab in patients with metastatic melanoma. N Engl J Med. 2010 Aug;363(8):71123.

5. Robert C, Schachter J, Long GV, Arance A, Grob JJ, Mortier L, et al.; KEYNOTE-006 investigators. Pembrolizumab versus ipilimumab in advanced melanoma. N Engl J Med. 2015 Jun;372(26):2521-32.

6. Larkin J, Chiarion-Sileni V, Gonzalez R, Grob JJ, Rutkowski P, Lao CD, et al. Five-Year Survival with Combined Nivolumab and Ipilimumab in Advanced Melanoma. N Engl J Med. 2019 Oct;381(16):1535-46.

7. Griffin M, Scotto D, Josephs DH, Mele S, Crescioli S, Bax HJ, et al. BRAF inhibitors: resistance and the promise of combination treatments for melanoma. Oncotarget. 2017 Aug;8(44):7817492.

8. Robert C, Grob JJ, Stroyakovskiy D, Karaszewska B, Hauschild A, Levchenko E, et al. Five-Year Outcomes with Dabrafenib plus Trametinib in Metastatic Melanoma. N Engl J Med. 2019 Aug;381(7):626-36.

9. Dummer R, Ascierto PA, Gogas HJ, Arance A, Mandala M, Liszkay $\mathrm{G}$, et al. Overall survival in patients with BRAF-mutant melanoma receiving encorafenib plus binimetinib versus vemurafenib or encorafenib (COLUMBUS): a multicentre, open-label, randomised, phase 3 trial. Lancet Oncol. 2018 Oct;19(10):1315-27.

10. Larkin J, Ascierto PA, Dréno B, Atkinson V, Liszkay G, Maio M, et al. Combined vemurafenib and cobimetinib in BRAF-mutated melanoma. N Engl J Med. 2014 Nov;371(20):1867-76.

11. Melichar B. Laboratory medicine and medical oncology: the tale of two Cinderellas. Clin Chem Lab Med. 2013 Jan;51(1):99112.

12. Flaherty KT, Infante JR, Daud A, Gonzalez R, Kefford RF, Sosman J, et al. Combined BRAF and MEK inhibition in melanoma with 
BRAF V600 mutations. N Engl J Med. 2012 Nov;367(18):1694703.

13. Long GV, Stroyakovskiy D, Gogas H, Levchenko E, de Braud F, Larkin J, et al. Combined BRAF and MEK inhibition versus BRAF inhibition alone in melanoma. N Engl J Med. 2014 Nov;371(20):1877-88.

14. Robert C, Karaszewska B, Schachter J, Rutkowski P, Mackiewicz A, Stroiakovski D, et al. Improved overall survival in melanoma with combined dabrafenib and trametinib. N Engl J Med. 2015 Jan;372(1):30-9.

15. Daud A, Tsai K. Management of Treatment-Related Adverse Events with Agents Targeting the MAPK Pathway in Patients with Metastatic Melanoma. Oncologist. 2017 Jul;22(7):823-33.

16. Vera J, Paludo J, Kottschade L, Brandt J, Yan Y, Block M, et al. Case series of dabrafenib-trametinib-induced pyrexia successfully treated with colchicine. Support Care Cancer. 2019 Oct;27(10):3869-75.

17. Lindsay JN, Barras M. Facing the challenges of new melanomatargeted therapies: treatment of severe fevers associated with dabrafenib/trametinib combination therapy. J Oncol Pharm Pract. 2015 Aug;21(4):293-5.

18. Menzies AM, Ashworth MT, Swann S, Kefford RF, Flaherty K, Weber J, et al. Characteristics of pyrexia in BRAFV600E/K metastatic melanoma patients treated with combined dabrafenib and trametinib in a phase I/II clinical trial. Ann Oncol. 2015 Feb;26(2):415-21.

19. Wachter H, Fuchs D, Hausen A, Reibnegger G, Werner ER. Neopterin as marker for activation of cellular immunity: immunologic basis and clinical application. Adv Clin Chem. 1989;27:81-141.

20. Melichar B, Spisarová M, Bartoušková M, Krčmová LK, Javorská L, Študentová H. Neopterin as a biomarker of immune response in cancer patients. Ann Transl Med. 2017 Jul;5(13):280.

21. Mura P, Barriere M, Papet $Y$, Reiss D, Camenen I, Vaillant L, et al. The clinical significance of urinary neopterin in the follow-up of patients after exeresis of a malignant melanoma. Pteridines. 1989;1(1):19-21.

22. Weinlich G, Murr C, Richardsen L, Winkler C, Fuchs D. Decreased serum tryptophan concentration predicts poor prognosis in malignant melanoma patients. Dermatology. 2007;214(1):8-14.

23. Zezulová M, Bartoušková M, Hlídková E, Adam T, Kujovská Krčmová L, Červinková B, et al. Citrulline as a biomarker of gastrointestinal toxicity in patients with rectal carcinoma treated with chemoradiation. Clin Chem Lab Med. 2016 Feb;54(2):305-14.

24. Sucher R, Schroecksnadel K, Weiss G, Margreiter R, Fuchs $D$, Brandacher G. Neopterin, a prognostic marker in human malignancies. Cancer Lett. 2010 Jan;287(1):13-22.

25. Sramek V, Melichar B, Indrakova J, Studentova H, Kalabova H, Vrana D, et al. Risk factors of atherosclerosis in patients with history of breast cancer. Pteridines. 2013;24(3):201-10.

26. Buchler T, Klapka R, Melichar B, Brabec P, Dusek L, Vyzula R, et al. Sunitinib followed by sorafenib or vice versa for metastatic renal cell carcinoma-data from the Czech registry. Ann Oncol. 2012 Feb;23(2):395-401.
27. Ravaud A, Barrios CH, Alekseev B, Tay MH, Agarwala SS, Yalcin S, et al. RECORD-2: phase II randomized study of everolimus and bevacizumab versus interferon $\alpha-2 a$ and bevacizumab as first-line therapy in patients with metastatic renal cell carcinoma. Ann Oncol. 2015 Jul;26(7):1378-84.

28. Melichar B, Gregor J, Solichová D, Lukes J, Tichý M, Pidrman V. Increased urinary neopterin in acute myocardial infarction. Clin Chem. 1994 Feb;40(2):338-9.

29. Melichar B, Solichová D, Melicharová K, Malírová E, Cermanová M, Zadák Z. Urinary neopterin in patients with advanced colorectal carcinoma. Int J Biol Markers. 2006 JulSep;21(3):190-8.

30. Solichova D, Melichar B, Blaha V, Klejna M, Vavrova J, Palicka $\mathrm{V}$, et al. Biochemical profile and survival in nonagenarians. Clin Biochem. 2001 Oct;34(7):563-9.

31. Melichar B, Jandik P, Krejsek J, Solichova D, Drahosova M, Skopec F, et al. Mitogen-induced lymphocyte proliferation and systemic immune activation in cancer patients. Tumori. 1996 May-Jun;82(3):218-20.

32. Melichar B, Nash MA, Lenzi R, Platsoucas CD, Freedman RS. Expression of costimulatory molecules CD80 and CD86 and their receptors CD28, CTLA-4 on malignant ascites CD3+ tumour-infiltrating lymphocytes (TIL) from patients with ovarian and other types of peritoneal carcinomatosis. Clin Exp Immunol. 2000 Jan;119(1):19-27.

33. Melichar B, Savary C, Kudelka AP, Verschraegen C, Kavanagh JJ, Edwards CL, et al. Lineage-negative human leukocyte antigen-DR+ cells with the phenotype of undifferentiated dendritic cells in patients with carcinoma of the abdomen and pelvis. Clin Cancer Res. 1998 Mar;4(3):799-809.

34. Melichar B, Tousková M, Solichová D, Králicková P, Kopecký G. CD4+ T-lymphocytopenia and systemic immune activation in patients with primary and secondary liver tumours. Scand J Clin Lab Invest. 2001;61(5):363-70.

35. Hanahan D, Weinberg RA. Hallmarks of cancer: the next generation. Cell. 2011 Mar;144(5):646-74.

36. Melichar B, Nemcová I. Eye complications of cetuximab therapy. Eur J Cancer Care (Engl). 2007 Sep;16(5):439-43.

37. Melichar $B$, Kohout $P$, Brátová $M$, Solichová $D$, Králícková $P$, Zadák Z. Intestinal permeability in patients with chemotherapyinduced stomatitis. J Cancer Res Clin Oncol. 2001 May;127(5):314-8. 